\title{
Contribution aux études des biocoenoses subtidales
}

\author{
Helena Gamulin-Brida, Giuseppe Giaccone et Stjepko Golubić \\ Institut de Biologie de l'Université de Zagreb, Yougoslavie, \\ Institut de Botanique, Palermo, Italie, \\ et \\ Department of Biology, Yale University, New Haven, U.S.A.
}

\begin{abstract}
Contribution to investigations in subtidal biocoenoses. Pollution of sea water in subtidal biotopes is a very important ecologic factor. It opens up new perspectives in studies concerned with the laws of general ecology as well as applied ecology; it has grown into a problem of increasing importance in all areas of tourism and recreation. Although studies of pollution effects on marine biocoenoses are still in their initial phase, they demonstrate the possibility of establishing the degree of pollution and its biological effects on the basis of qualitative and quantitative investigations of benthic fauna and flora. Experience in this field is still quite scant in comparison to that gained about systems of biologic indications in fresh waters, which have been the subject of numerous studies for years. We are well aware that to approach the problem on the level of biocoenoses implies a great many complex difficulties, yet we consider the study of biocoenoses a suitable basis for obtaining better insights into the equally complex effects of polluted waters, which lead to disturbances of the natural balance. The number of species which respond to slight changes in the chemistry of sea water and hence could be used as direct indicators ("Leitformen") is very small. Quantitative changes in individual species due to pollution and shifting of the equilibrium within biocoenoses could serve as sensitive indications of the influence of waste waters. Generally, marine biocoenoses react to the inflow of waste according to the basic ecologic law for extreme biotopes: reduction in the number of species in conjunction with increase in the number of individuals. In special cases some specific characteristics may occur.
\end{abstract}

\section{INTRODUCTION}

L'étude des biocoenoses benthiques du bassin de l'Adriatique moyenne (GAmulinBRIDA 1962, 1965a) a permis d'établir l'existence de deux entités écologiques bien marquées qui influent de façon notable sur la structure et la distribution des biocoenoses: la zone côtière soumise à l'influence très forte du continent et des eaux continentales, et la zone de la haute mer. ERCEGović (1964), en ce qui concerne la répartition horizontale de la végétation des Algues en Adriatique, distingue la végétation des Algues des eaux extérieures et des eaux côtières en analysant en détails ce phénomène et ses causes. Dans la zone des eaux pures extérieures les peuplements coralligènes, ainsi que la végétation des Algues en général, et surtout des Corallinaceae, sont beaucoup mieux développés que dans la zone côtière; entre autres causes interviennent les différences de régime thermohalin et les courants: ErcEgović (1964) a 
constaté l'influence de la sédimentation, notablement plus forte et plus rapide dans les eaux côtières que dans la zone de la haute mer.

Dans la zone côtière, dans les ports plus importants (Split, Šibenic etc.) entre aussi en ligne de compte le facteur «pollution des eaux marines». Ce facteur a été étudié à part, en ce qui concerne la végétation des Algues dans la région de l'Adriatique septentrionale près de Rovinj (Golubić, sous presse).

L'expérience que nous avons acquise au cours de nos recherches sur les biocoenoses benthiques et l'action des eaux polluées en Adriatique, nous l'avons utilisée dans notre étude sur les conditions biocoenotiques en mer Tyrrhénienne, près de Naples. Cette communication se rapporte principalement à ces récentes recherches.

$\mathrm{Au}$ cours de nos recherches sur les biocoenoses benthiques dans le Golfe de Naples (GAMulin-Brida 1965b) et aux alentours de Palermo (Giaccone 1965), nous avons constaté dans une partie de ces régions l'influence notable des eaux polluées sur les peuplements benthiques. La vaste région du Golfe de Naples, entre autres, englobe des biotopes qui se ressemblent et ne se différencient guère que par le degré de pollution des eaux. Le but de nos recherches était d'étudier ces différences entre les peuplements benthiques, dues à l'influence du facteur de la pollution des eaux. Dans le cas qui nous occupe il s'agit surtout de la pollution de l'eau par les canalisations de la ville (les localités explorées sont loin du port pour les bateaux de fort tonnage et des deversements des résidus des usines).

Le problème de la pollution des eaux marines fait actuellement, l'objet de nombreuses recherches et le thème de congrès scientifiques ${ }^{1}$.

Pourtant les recherches biologiques de l'indice de qualité de l'eau de mer n'en sont encore qu' à leur phase initiale comparées aux systèmes d'indication biologique de la qualité des eaux douces qui font, depuis des années, l'objet de nombreuses études.

\section{MÉTHODES}

Nous avons employé la méthode phytosociologique de Braun-Blanquet (1951), modifiée par Molinier \& Molinier (1955) et par Peres \& PiCard $(1958,1964)$ pour la bionomie marine. On a exécuté des relevés similaires à ceux de l'Ecole zuricho-montpélliéraine, indiquant les coefficients d'abondance-dominance et de sociabilité. Les prélèvements directs étaient effectués au moyen du scaphandre autonome (diving techniques), le long d'une corde calibreé, en largeur d'un metre. Ainsi la manière de prélèvements sur les surfaces carrées était combinée avec celle de transsections.

Une méthode semblable était utilisée dans les études des biocoenoses benthiques de la baie de Porto Paone près de Naples (Gamulin-Brida 1965) et des phytocoenoses marines des alentours de la Sicile (De Leo \& Giaccone 1964, Giaccone \& Sortino 1964, Giaccone 1965). Pendant nos recherches sur les biocoenoses circalittorales de l'Adriatique moyenne (GAMULIN-BRIDA 1962) nous avons procédé à une comparaison

I Second International Conference on Water pollution Research, Tokio, 24-28 août 1964; Symposium International des Pollutions marines, Monaco, 31 mars au 4 avril 1964, etc. La Commission Internationale pour l'Exploration Scientifique de la Mer Méditerranée comprend un Comité spécial de Microbiologie, de Microchimie et des Pollutions marines. 
des résultats obtenus par la méthode statistique de SORENSEN (1948), originelle et modifiée, avec ceux des méthodes sociologiques pré-citées; on constate la concordance des résultats obtenus par ces méthodes différentes.

\section{EXPOSÉ ANALYTIQUE DES PEUPLEMENTS PROSPECTÉS}

Dans la région du Golfe de Naples nous avons tracé deux profils, tous les deux sur le fond d'origine volcanique, de pente relativement douce et de mode modérement exposé aux vagues. Un profil est situé sur la côte externe, sud-occidentale, de l'île de Procida, baignée par des eaux vierges venant du large de la mer Tyrrhénienne; l'autre près du Cap Posillipo dans la partie intérieure, nord-occidentale, du Golfe, soumise aux cours des eaux polluées du port. Les profils contiennent pour la plupart des fonds rocheux, partiellement des graviers et des sables, parcourant aussi les biocoenoses de l'herbier de Posidonies et de la pelouse de Cymodocées, jusq'à une profondeur d'env. $30 \mathrm{~m}$. Nous avons établi encore une station dans les eaux plus fortement polluées, sur le petit môle de Mergelina, dans la partie plus abrité du Golfe. Une comparaison de la végétation des fonds calcaires, se trouvant dans des eaux polluées à divers degrés, était effectuées dans l'Adriatique nord, aux environs de Rovinj (Golubić, in press).

$$
\begin{gathered}
\text { Profil des eaux pures, ile de Procida } \\
\text { (longuer du profil } 90 \mathrm{~m} \text {, profondeur a } 30 \mathrm{~m} \text { ) }
\end{gathered}
$$

Etage supralittoral. La biocoenose de la roche supralittorale est très bien developpée et représentée par le mềme peuplement que sur les côtes calcaires méditerranéennes, sauf les Cyanophycées endolithes qu'on ne trouve pas sur ces roches volcaniques. L'espèce caractéristique, Littorina (Melaraphe) neritoides est abondante, surtout dans les petits enfoncements et sur les parties accidentées de la roche; Ligia italica est aussi nombreuse. Vers le bas de la roche supralittorale on trouve de petites colonies de Chthamalus depressus surtout dans les microlocalités mouillées par l'écoulement des vagues brisées.

Etage médiolittoral. Tout comme les roches supralittorales les roches médiolittorales sont recouvertes par des Cyanophycées épilithes. On peut bien distinguer deux biocoenoses, cependant les petites sphérules noirâtres de l'algue Rivularia atra se trouvent, sur cette localité, non seulement dans l'horizon inférieur, mais aussi sur la roche de l'horizon superieur de l'étage médiolittoral, parmi les nombreux individus de Chtbamalus stellatus. Tous les autres espèces présentent la répartition classique, connue sur la plupart des côtes de la mer Méditerranée (Peres \& Picard 1964).

Voici le relevé bionomique (Tableaux 1 et 2 , Figure 1 ).

La biocoenose de la roche médiolittorale inférieure, dans cette localité, se distingue, outre par ses espèces caractéristiques, aussi par les amas de Brachiodontes minimus et par les petits buissons de Laurencia papillosa avec Melobésiées épiphytes. Tous les deux espèces, comme aussi Pachygrapsus marmoratus, Actinia equina et Monodonta turbinata sont fréquentes dans l'horizon superficiel de l'étage infralittoral. 


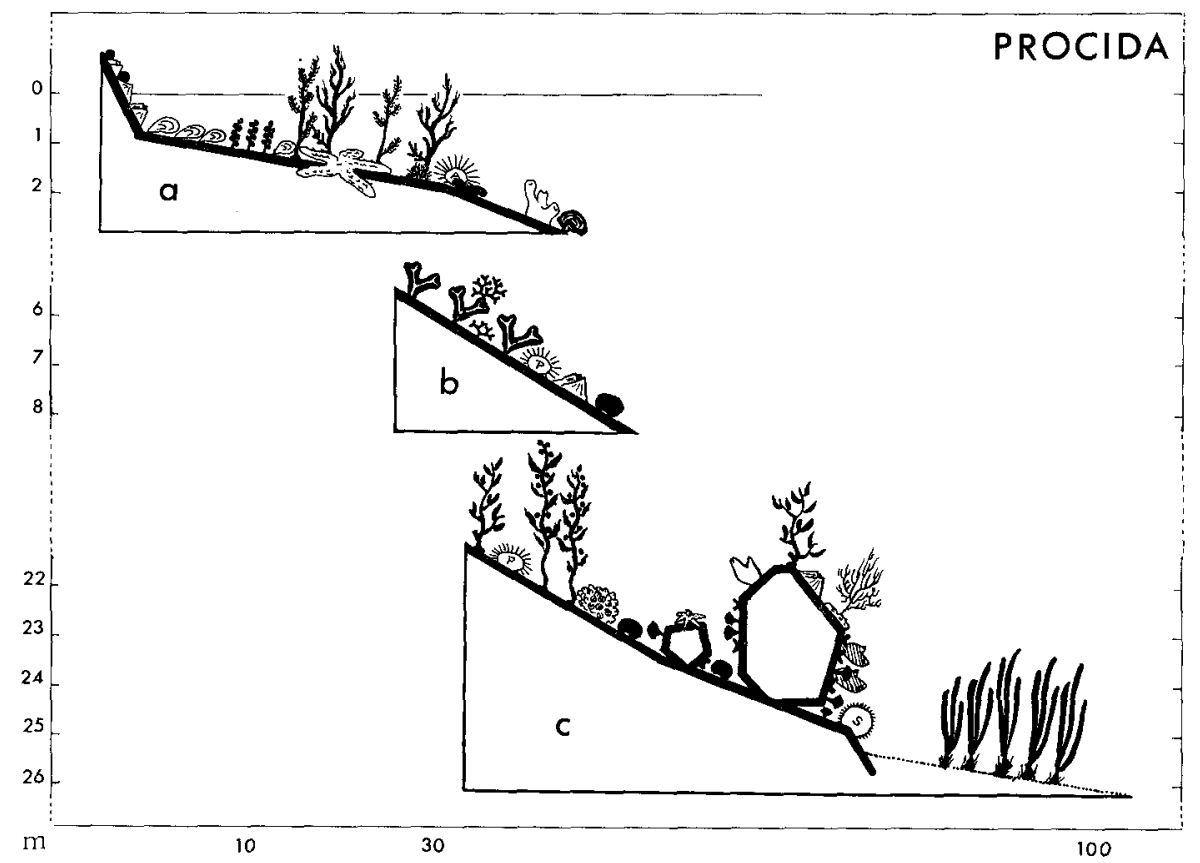

Fig. 1: Peuplements des eaux pures, oligotrophes, le long du profil «Procida». (a) Rivularia atra, Cbthamalus stellatus, Patella lusitanica, Balanus perforatus, Brachyodontes minimus, Laurencia papillosa, Cystoseira mediterranea, C. abrotanifolia, Marthasterias glacialis, Jania rubens, Arbacia lixula, Lithothamnion lenormandi, Verongia aerophoba, Padina pavonia. (b) Dictyopteris membranacea, Dictyota dichotoma, Paracentrotus lividus, Codium bursa. (c) Cystoseira discors, Sargassum linifolium, Cladocora cespitosa, Udotea desfontainii, Echinaster sepositus, Peyssonnelia squamaria, Halocyntbia papillosa, Eunicella cavolini, Schizoporella sanguinea, Microcosmus sulcatus, Spbaerechinus granularis, Posidonia oceanica

Etage infralittoral. Le fond rocheux s'étend jusqu'à $15 \mathrm{~m}$; puis les grandes roches isolées suivent, séparées l'une de l'autre par des canaux de sable. Au fur et à mesure qu'on s'éloigne de la côte les canaux de sable deviennent plus larges, et les roches émergeant des sédiment sableux plus rares; puis on ne trouve plus que quelques blocs et pierres gisant sur le sable; à partir de $25 \mathrm{~m}$ de profondeur la base rocheuse est entièrement ensevelie sous les sédiments sableux, peuplés par l'herbier de Posidonies.

Tableau 1

Horizon supérieur de l'étage médiolittoral

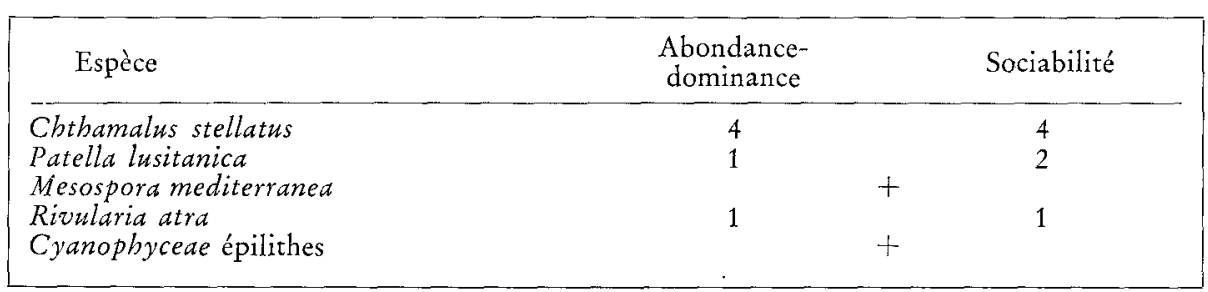


Tableau 2

Horizon inférieur de l'étage médiolittoral

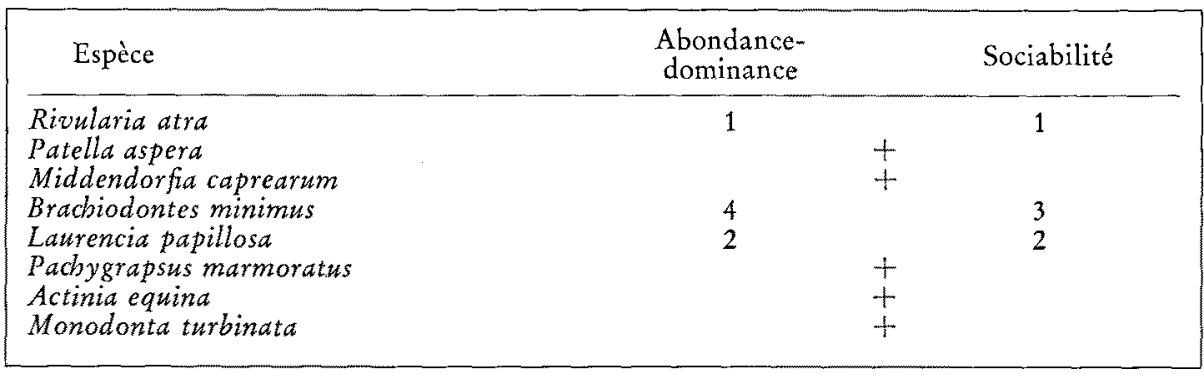

Sur le fond rocheux la biocoenose des algues photophiles est très bien developpée. Entre la surface et le fonds on peut distinguer plusieurs zones: Zone à Laurencia papillosa.

Bien que dans l'étage médiolittoral on trouve quelques buissons de cette Rhodophycée elle ne forme une zone dense qu'au commencement de l'étage infralittoral (limité vers le haut par le niveau inférieur des émersions normales et vers le bas par la limite inférieure des Zostéracées) jusqu’à env. $80 \mathrm{~cm}$ de profondeur (Tableau 3):

Tableau 3

Zone à Laurencia papillosa

\begin{tabular}{|c|c|c|}
\hline Espèce & $\begin{array}{l}\text { Abondance- } \\
\text { dominance }\end{array}$ & Sociabilité \\
\hline Laurentia papillosa & 4 & 4 \\
\hline Brachyodontes minimus & 3 & 4 \\
\hline Actinia equina & 1 & 1 \\
\hline Monodonta turbinata & 1 & 1 \\
\hline Pacbygrapsus marmoratus & & \\
\hline Patella coerulea & & \\
\hline Balanus perforatus & 2 & 2 \\
\hline
\end{tabular}

Tandis que dans la zone superficielle à Laurencia papillosa on trouve des espèces qui remontent sur les roches médiolittorales et supportent aisement des émersions limitées, la zone suivante à Cystoseira mediterranea ne contient que des espèces purement infralittorales (Tableau 4).

Certaines espèces qui étaient abondantes dans la zone superficielle ici, dans la zone à Cystoseira mediterranea, se raréfient, par exemple Laurentia papillosa, et Balanus perforatus, d'autres deviennent plus fréquentes, telles que Hymeniacidon sanguinea formant des incrustations (oranges, blanchâtres) sur les roches, et Jania rubens, mêlée à d'autres espèces gazonnantes, plus rare en épiphyte. Cystoseira mediterranea et C.abrotanifolia sont répandues exclusivement dans cette zone, tandis que des espèces telles que Dictyopteris membranacea, Sargassum linifolium, Verongia aeropboba, Paracentrotus lividus, Echinaster sepositus, commençent ici et deviennent plus abondantes dans les zones suivantes. Il y a des nombreux épibiontes surtout sur les «branches» de deux 
Tableau 4

Zone à Cystoseira mediterranea (de 0,8 à $2 \mathrm{~m}$ )

\begin{tabular}{|lcc|}
\hline \multicolumn{1}{|c}{ Espèce } & $\begin{array}{c}\text { Abondance- } \\
\text { dominance }\end{array}$ & Sociabilité \\
\hline Cystoseira mediterranea & 3 & 2 \\
Cystoseira abrotanifolia & 2 & 2 \\
Ectocarpus paradoxus & $2 \mathrm{e}$ & 2 \\
Padina paronia & 1 & 2 \\
Dictyopteris membranacea & 1 & 2 \\
Jania rubens & 1 & 1 \\
Dictyota dichotoma & 1 & 2 \\
Balanus perforatus & 1 & 2 \\
Arbacia lixula & 1 & 1 \\
Marthasterias glacialis & 1 & 1 \\
Hymeniacidon sanguinea & 1 & \\
\hline
\end{tabular}

Cystoseires: Ectocarpus paradoxus, diverses Melobesiae, Ophiotbrix fragilis, Clatbrina coriacea, etc. Ce milieu oligotrophique contient un nombre énorme d'espèces, representées par une quantité faible des individus: Patella coerulea, Cerithium rupestre, C. vulgatum, Pisania maculosa, Anemonia sulcata, Murex trunculus, Acantbonyx lunulatus, Eriphia spinifrons, diverses Pagurides, Holotburia tubulosa, Amphiroa rigida, Colpomenia sinuosa, Dictyota linearis, Anadyomene stellata et diverses autres espèces accompagnatrices.

A la profondeure de 2 à $6 \mathrm{~m}$ la très forte influence des vagues empêche le développement de la végétation des algues arborescentes; les roches, d'apparence nues, sont cependant partiellement recouvertes par des Melobesiées surtout d'une couche violâtre-blanchâtre de Litbothamnium lenormandi, et localement par des incrustations d'Eponges, surtout de Hymeniacidon sanguinea (Tableau 5):

Tableau 5

Zone à Lithothamnium lenormandi (de 2 ì $6 \mathrm{~m}$ )

\begin{tabular}{|c|c|c|}
\hline Espèce & $\begin{array}{l}\text { Abondance- } \\
\text { dominance }\end{array}$ & Sociabilité \\
\hline Lithothamnium lenormandi & 4 & 3 \\
\hline Hymeniacidon sanguinea & 2 & 2 \\
\hline Verongia aerophoba & 2 & 2 \\
\hline Padina pavania & $i$ & 2 \\
\hline Paracentrotus lividus & 2 & 1 \\
\hline Arbacia lixula & & \\
\hline
\end{tabular}

Cladocora cespitosa forme ça et là des petites colonies (diametre env. $5 \mathrm{~cm}$ ) et plus profondement construit de grands amas (diametre env. 20-50 cm). Murex trunculus rampe sur les roches; Marthasterias glacialis et Ophioderma longicauda se logent dans les enfoncements des roches; il y a aussi quelques exemplaires de Codium bursa et Dilophus ligulatus.

Le fond rocheux plus profond est caractérisé par la dominance de Dictyopteris membranacea (Tableau 6): 
Tableau 6

Zone à Dictyopteris membranacea (de 6 à $10 \mathrm{~m}$ )

\begin{tabular}{|c|c|c|c|}
\hline Espèce & $\begin{array}{l}\text { Abondance- } \\
\text { dominance }\end{array}$ & & Sociabilité \\
\hline Diciyopteris membranacea & 3 & & 2 \\
\hline Dictyota dichotoma & 1 & & 2 \\
\hline Cystoseira discors $\mathrm{f}$. tenuiramosa & 1 & & 2 \\
\hline Codium bursa & 1 & & 2 \\
\hline Codium difforme & & + & \\
\hline Sargassum linifolium & & + & \\
\hline Paracentrotus lividus & 1 & & 1 \\
\hline Arbacia lixula & & + & \\
\hline Balanus perforatus & 1 & & 1 \\
\hline
\end{tabular}

Sur les colonies de Balanus perforatus on trouve le Bryozoaire Schizoporella sanguinea, l'Eponge Timea unistellata et divers autres épibiontes; Polysiphonia setigera est fréquente comme épiphyte sur les autres algues. Les espèces animales, dont quelques-unes sont citées dans les zones à Cystoseira mediterranea et à Lithothamnium lenormandi, sont présentes dans tout le biotope de la tiocoenose des algues photophiles, quoique en quantité très modérée.

Après $10 \mathrm{~m}$ de profondeur les sédiment sableux s'accumulent dans les enfoncements, et alors les roches sont séparées par des canaux sableux; la biocoenose des algues photophiles persiste sur la partie éclairée des roches, de blocs et des pierres, tandis que des élements sciaphiles, précoralligènes et coralligènes s'installent vers la base et sur les parois ombragées des roches; les Posidonies occupent les couches sableuses, stabilisées et suffisement épaisses et humifiées.

Peuplement des parties rocheuses éclairées (de 10 à $20 \mathrm{~m}$ ): La lumière change, naturellement, en qualité et en quantité, en fonction de la profondeur; cependant le peuplement des sommets et des autres parties éclairées des roches et des pierres appartiement à la biocoenose des algues photophiles, selon Peres \& Picard $(1958,1964)$, où

\section{Tableau 7}

Peuplement des parties éclairées (de 10 à $20 \mathrm{~m}$ )

\begin{tabular}{|c|c|c|}
\hline Espèce & $\begin{array}{l}\text { Abondance- } \\
\text { dominance }\end{array}$ & Sociabilité \\
\hline Cystoseira discors f. latiramosa & 3 & 3 \\
\hline Cystoseira discors f. tenuiramosa & 1 & 2 \\
\hline Dictyopteris membranacea & 2 & 2 \\
\hline Sargassum linifolium & 2 & 2 \\
\hline Halopteris filicina & $2 \mathrm{e}$ & \\
\hline Paracentrotus lividus & 2 & 2 \\
\hline Arbacia lixula & & \\
\hline Verongia aeropboba & & \\
\hline Balanus perforatus & 1 & 2 \\
\hline Echinaster sepositus & 1 & 1 \\
\hline Marthasterias glacialis & & \\
\hline Murex trunculus & & \\
\hline
\end{tabular}


on prend le terme-«photophilie» dans un sens large contenant toute une échelle des espèces quant à leur exigence en regard de la gradation d'éclairement. Sur ces localités relativement éclairées le peuplement animal est le même que dans les zones précédentes - le fait démontrant la continuité de la même biocoenose -; le peuplement algal est semblable à celui de la zone à Dictyopteris membranacea, mais l'élement exigeant

Tableau 8

Peuplement des partie rocheuses ombragées (de 10 à $20 \mathrm{~m}$ )

\begin{tabular}{|c|c|c|c|}
\hline Espèce & $\begin{array}{l}\text { Abondance- } \\
\text { dominance }\end{array}$ & & Sociabilité \\
\hline \multicolumn{4}{|l|}{ (a) Peuplement de l'aspect précoralligène: } \\
\hline Peyssonnelia squamaria & 2 & & $3 \mathrm{e}$ \\
\hline Peyssonnelia rubra & 1 & & 2 \\
\hline Peyssonnelia polymorpha & & + & \\
\hline Litbophyllum incrustans & 1 & & 2 \\
\hline Codium difforme & 1 & & 2 \\
\hline Codium bursa & 2 & & 3 \\
\hline Udotea desfontainii & 1 & & 3 \\
\hline Halimeda tuna & & + & \\
\hline Cladocora cespitosa & 2 & & 2 \\
\hline Eunicella cavolini & & + & \\
\hline Chondrilla nucula & 1 & & 1 \\
\hline Ostrea edulis & 1 & & 2 \\
\hline Microcosmus sulcatus & 2 & & 3 \\
\hline Botrillus schlosseri & 1 & & 1 \\
\hline Halocynthia papillosa & 1 & & 1 \\
\hline Schizoporella sanguinea & 1 & & 1 \\
\hline Antedon mediterranea & & + & \\
\hline Sphaerechinus granularis & & + & \\
\hline \multicolumn{4}{|l|}{ (b) Peuplement des endroits bien ombragés: } \\
\hline Pseudolithophyllum expansum $\mathrm{f}$. stictaeformis & 1 & & 2 \\
\hline Pseudolitbophyllum expansum f. genuina & & + & \\
\hline Lithothamnium philipii & 1 & & 2 \\
\hline Hacelia attenuata & 1 & & 1 \\
\hline Verongia cavernicola & 1 & & 1 \\
\hline Chondrosia reniformis & 2 & & 3 \\
\hline Petrosia ficiformis & 1 & & 1 \\
\hline Crambe crambe & & + & \\
\hline Axinella damicornis & & + & \\
\hline Cliona viridis & & + & \\
\hline Oscarella lobularis & & + & \\
\hline Lissa chiragra & & + & \\
\hline Dromia vulgaris avec Didemnum maculosum & & + & \\
\hline Eunicella cavolini avec Microciona sp. & & + & \\
\hline Schizoporella sanguinea & 2 & & 2 \\
\hline Myriapora truncata & & + & \\
\hline Porella cervicornis & & + & \\
\hline Balanophyllia italica & & + & \\
\hline Cladocora cespitosa & 2 & & 2 \\
\hline Halocyntbia papillosa & 2 & & 2 \\
\hline Microcosmus sulcatus & 2 & & 2 \\
\hline Botryllus scblosseri & 2 & & 2 \\
\hline Didemnum maculosum & & + & \\
\hline Antedon mediterranea & & + & \\
\hline Serpula vermicularis & & + & \\
\hline
\end{tabular}


une diminution d'éclairement augmente, par exemple Sargassum linifolium; l'espèce dominante est Cystoseira discors f. latiramosa (Tableau 7).

La macrofaune en épibiose est qualitativement la même que dans les zones précédentes, cependant la quantité de Rissoides et d'Ophiures sur les Cystoseires augmente. Parmi les nombreux épiphytes la Phaeophycé Halopteris filicina est la plus abondante; les espèces Thuretella schousboei, Dictyota linearis, Cladophora pellucida, Polysiphonia tenerrima et Polysiphonia flexella sont aussi fréquentes.

Peuplement des partie rocheuses ombragée (de 10 à $20 \mathrm{~m}$ ): C'est la biocoenose coralligène qui peuple les parties rocheuses ombragées; cependant l'aspect «précoralligène» prédomine et le coralligène d'horizon inférieur de la roche littorale (Peres \& PiCARD 1964) n'est devéloppé qu'à la base des roches et sur les parois le plus ombragés (Tableau 8).

Il y a de nombreuses espèces, représentées par quelques individus, partout sur le fond rocheux, par exemple Murex trunculus, Tritonalia erinacea, Fusus syracusanus, Cerithium vulgatum, Haliotis lamellosa, Chiton olivaceus, Spirographis spallanzanii. Les épibiontes sont très nombreux surtout sur les récifs morts de Cladocora cespitosa (Cistella cuneata, Chama gryphoides, Serpula vermicularis), sur Microcosmus sulcatus, sur diverses coquilles ainsi que sur les crabes, tels que Pisa armata, Maia verrucosa etc.; par exemple, la Synascidie Didemnum maculosum sur le dos de Dromia vulgaris, les Eponges Oscarella lobularis sur les valves d'Ostrea edulis et Microciona gradalis sur la coquille de Murex trunculus. SARA \& SiRIBelli (1960) ont entrepris des études speciales sur les Eponges de cette région. Comme dans les zones déjà traitées, il y a aussi ici un grand nombre d'espèces, pauvres en individus.

La biocoenose de l'herbier de Posidonies, qui commence dans les canaux sableux, devient luxuriante et très dense sur le vaste biotope sablo-vaseux de 25 à $30 \mathrm{~m}$ de profondeur.

Dans tous les biotopes de ce profil prospecté nous avons rencontré aussi des poissons, à espèces variées, mais représentées par des individus peu nombreux.

\section{Profil des eaux modérément polluées, eutrophes, Cap de Posillipo$$
\text { (longueur du profil } 100 \mathrm{~m} \text {, profondeur o à } 20 \mathrm{~m} \text { ) }
$$

Pour éviter les répétitions nous n’exposerons ici que les différences entre les deux profils prospectés.

Une difference évidente se voit dans la biocoenose de la roche médiolittorale supérieure: le peuplement du Cirripède Chthamalus stellatus est moins abondant et partiellement remplacé par l'Algue Chatomorpha tortuosa; Rivularia atra ne se trouve que dans la biocoenose de la roche médiolittorale inférieure, en quantité très faible (Fig. 2).

Au lieu de colonies de Brachiodontes minimus, sur le profil de Cap Posillipo, on trouve des populations denses de Mytilus galloprovincialis, dans la biocoenose de la roche médiolittorale inférieure ainsi que dans l'horizon superficiel de l'étage infralittoral. Dans le biotope des algues photophiles on ne trouve pas les zones à Laurencia pa- 


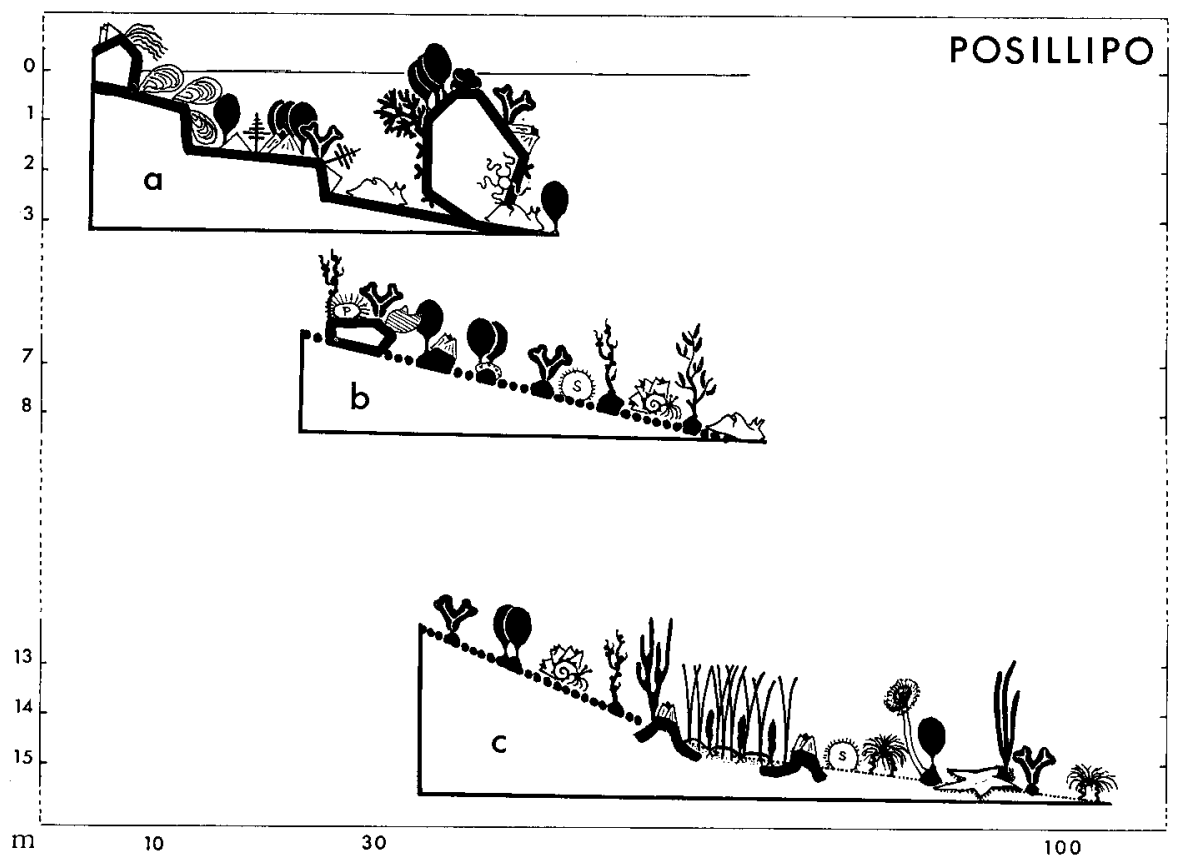

Fig. 2: Peuplements des eaux légèrement polluées, eutrophes, le long du profil «Posillipo». (a) Chthamalus stellatus, Chaetomorpha tortuosa, Mytilus galloprovincialis, Ulva lactuca, Patella aspera, Pterocladia capillacea, Balanus perforatus, Dictyopteris membranacea, Aplisia fasciata, Peyssonnelia squamaria, Codium tomentosum, Colpomenia sinuosa, Ophioderma longicauda. (b) Gracillaria confervoides, Paracentrotus lividus, Microcosmus sulcatus, Schizoporella sanguinea, Sphaerechinus granularis, Pagurides, Cystoseira discors. (c) Codium elongatum, Cymodocea nodosa, Caulerpa prolifera, Ceriantbus membranacetss, Spirographis spalanzanii, Astropecten aurantiacus, Posidonia oceanica

pillosa ni à Cystoseira mediterranea, lesquelles sont remplacées par une abondante végétation moins riche en espèces: Ulva lactuca et Pterocladia capillacea prédominent, Aplysia fasciata, $A$. depilans et Balanus perforatus parmi les animaux. Il y a beaucoup de grands exemplaires d'Aplysia fasciata qui se couchent parmi les Ulves. Patella aspera et Opbioderma longicauda, sont beaucoup plus nombreuses que sur le profil de l'île de Procida; il y a aussi Codium tomentosum et Colpomenia sinuosa aux bords des rochers, et vers la base, dans les parties ombragées, Peyssonnelia squamaria. Dictyopteris membranacea est très repandue le long du profil du Cap Posillipo, quoique cette Algue ne forme pas ici une zone distincte comme sur le profil de l'île de Procida (Tableau 9).

Sur les roches profondes de 3 à $6 \mathrm{~m}$ les espèces dominantes sont Hypnea musciformis, indicatrice des eaux polluées, et Dictyopteris membranacea, Phaeophycée généralement très répandue dans le Golfe de Naples, indiquant une diminution de l'éclairement et, peut-être, la présence des eaux plus ou moins souillées (d'après Pérès \& Picard 1958).

Après $8 \mathrm{~m}$ de profondeur les roches sont séparées par des canaux sableux et 
Tableau 9

Profil Posillipo (étage médiolittoral: a, b; étage infralittoral: $c, d, e$ )

Espèce

Abondancedominance

Sociabilité

(a) Biocoenose de la roche médiolittorale supérieure:

Chthamalus stellatus

Chaetomorpha tortuosa

Patella lusitanica

1

Mesospora mediterranea

Cyanopbyceae épilithes

+
$\quad$
$\quad$
$\quad+$
+

(b) Biocoenose de la roche médiolittorale inférieure:

Mytilus galloprovincialis

Balanus perforatus

4

Middendorfia caprearum

Ulva lactuca

Pterocladia capillacea

Patella aspera

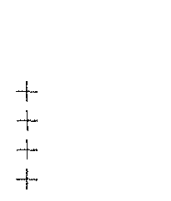

(c) Biocoenose des algues photophiles (de 30 à $100 \mathrm{~cm}$ ):

Balanus perforatus

Monodonta turbinata

Patella aspera

Patella coermlea

Mytilus galloprovincialis

Ulva lactuca

Pterocladia capillacea

Dictyopteris membranacea

2

2

(d) Au centre des roches (de 1 ì $3 \mathrm{~m}$ ):

Ulva lactuca

Codium tomentosum

Colpomenia sinuosa

$\begin{array}{ll}4 & 4 \\ 1 & + \\ 1 & 1 \\ 2 & 1 \\ 2 & 3 \\ 3 & 3 \\ 2 & 3 \\ & \end{array}$

(e) Au bord des roches:

Dictyopteris membranacea

Aplysia fasciata

Aplysia depilans

Hypnea musciformis

Peyssonnelia squamaria

Opbioderma longicauda

après $10 \mathrm{~m}$ de profondeur on trouve des roches et des pierres isolées sur le fond sableux. Localement, dans les profondeurs de 3 à $6 \mathrm{~m}$, ainsi que plus profondement, jusqu'à $12 \mathrm{~m}$, il y a quelques rochers formant des surplombs. Les sommets ainsi que les bords des rochers sont peuplés par les mêmes espèces, citées ci-dessus; il y a aussi quelques exemplaires de Paracentrotus lividus.

Sous les surplombs on trouve un peuplement sciaphile de l'aspect précoralligène; la biocoenose coralligène typique n'est pas développée sur ce profil de Cap Posillipo (Tableau 10).

Autour des roches, sur le fond sableux il y a une énorme quantité de Pagurides, dans les diverses coquilles, le plus fréquemment de Murex trunculus, portant très souvent des colonies de Balanus perforatus, partiellement recouvertes du Bryozoaire Scbizoporella sanguinea. Sur le sable on trouve aussi quelques exemplaires de Sphaerechinus 
Tableau 10

Biocoenose sous les surplombs des roches (de 3 à $12 \mathrm{~m}$ )

\begin{tabular}{|c|c|c|c|}
\hline Espèce & $\begin{array}{l}\text { Abondance- } \\
\text { dominance }\end{array}$ & & Sociabilité \\
\hline Balanus perforatus & 4 & & 4 \\
\hline Udotea desfontaini avec Melobesiae & 1 & & 1 \\
\hline Peyssonnelia squamaria & 2 & & 3 \\
\hline Peyssonnelia polymorpha & 1 & & 2 \\
\hline Litbotbamnion philippi & 1 & & 2 \\
\hline L. lenormandii & 1 & & 2 \\
\hline Schizoporella sanguinea & 3 & & 3 \\
\hline Didemnum macullosum & 2 & & 2 \\
\hline Ectyon oroides & & + & \\
\hline Zanardinia collaris & 1 & & 2 \\
\hline Bornetia secundiflora & & + & \\
\hline Cerithium vulgatum & 1 & & 1 \\
\hline Paracentrotus lividus & 2 & & 1 \\
\hline Ophioderma longicauda & 1 & & $i$ \\
\hline $\begin{array}{l}\text { Styela plicata } \\
\text { Microcosmus sulcatus }\end{array}$ & 1 & + & 1 \\
\hline
\end{tabular}

granularis et de nombreuses Holothuria tubulosa et $H$.polii. Sur tout le biotope, jusqu'à $14 \mathrm{~m}$ de profondeur il y a des pierres gisant çà et là sur le sable, portant des Algues, Gracillaria confervoides, Dictyopteris membranacea et Ulva lactuca, ainsi que quelques individus de Paracentrotus lividus, Microcosmus sulcatus, des colonies de Balanus perforatus et des incrustations de Schizoporella sanguinea. Sur le sédiment sableux fixé sur les roches par les Algues s'installe le peuplement de la pelouse à Cymodocea nodosa et Caulerpa prolifera, auparavant rare, devenant plus dense sur les couches sableuses plus vastes et plus épaisses. Parmi les Cymodocées, comme aussi dans le sable vide, Cerianthus membranaceus et Astropecten aurantiacus sont frequents, et, sur les pierres, Spirographis spallanzanii, Codium elongatum, Dictyopteris membranacea et Ulva lactuca.

Commençant à $14 \mathrm{~m}$ de profondeur, sur les sédiments sableux bien stabilisés et suffisament humifiés, s'installe la biocoenose de l'herbier à Posidonies. Cependant sur ce profil du Cap Posillipo l'herbier à Posidonies n'atteint pas la densité qu'il a dans les eaux pures du profil de l'île de Procida.

\section{Peuplement des eaux plus fortement polluées - môle et jetée des canaux "Mergelina»}

Les peuplements des étages supralittoral et médiolittoral sont les mêmes que sur le profil Posillipo; tout l'éspace qui correspond à l'infralittoral supérieur est occupé par des colonies très denses de Mytilus galloprovincialis et de Balanus perforatus voisinant avec les bryozoaires Bugula neritina et Zoobotryon verticillatum et l'hydrozoaire Sertularia abietina.

Les epiphytes sur les Mytilus et les Balanus sont des algues connues comme indicatrices des eaux polluées (Giaccone 1965): Chatomorpha tortuosa, Ectocarpus 
abreviatus, Bryopsis disticha, Ulva lactuca, Hypnea musciformis et Callithamnion scopolorum. Localement, il n'y a qu'une végétation dense de Ulva lactuca et Hypnea musciformis. Y succédent des sables et de la vase sans épibiontes visibles; au contraire dans le substrat on trouve des nombreux Polychètes, Nematodes etc., et, surtout, une riche microfaune.

\section{DISCUSSION}

Le profil «Procida» dans la région des eaux pures «oligotrophiques» montre des biocoenoses typiquement développées en allant de l'étage supralittoral jusqu'à la fin du profil dans la région de l'herbier à Posidonies. Dans la biocoenose des algues photophiles ressortent des zones caracterisées par la prédominance de certaines espèces. Les eaux oligotrophiques de cette région sont favorables à un grand nombre d'espèces representées par un petit nombre d'individus (à cause d'un faible taux de sels nutritifs). Le profil «Posillipo» dans la région des eaux légèrement polluées, «eaux eutrophiques», comprend les biocoenoses correspondant à celles du profil «Procida», mais l'équilibre des biocoenoses se déplace: les espèces communes à ces deux profils différent en degré d'abondance, ou, bien, sont remplacées par d'autres espèces. C'est ainsi que dans la biocoenose de la roche médiolittorale inférieure et dans l'horizon supérieur de la biocoenose des algues photophiles on remarque des colonies denses de Mytilus galloprovincialis et de Balanus perforatus. Dans la biocoenose des algues photophiles, sur le profil "Posillipo», on peut distinguer des zones differentes; des élements délicats des eaux pures oligotrophiques, tels que Cystoseira barbata et C. mediterranea disparaissent et font place à des espèces préferant les eaux eutrophiques, telles que Ulva lactuca, Pterocladia capillacea, Hypnea musciformis, Aplysia fasciata, A. depilans. Les espèces Ulva lactuca et Balanus perforatus sont représentées par un nombre particulièrement important d'exemplaires. La biocoenose de l'herbier à Posidonies est mieux développée dans la région des eaux pures de l'île de Procida que dans les eaux eutrophiques du Posillipo; l'herbier à Posidonies sur le profil de «Posillipo», est remplacé en partie par un peuplement de Cymodocées, ce qui indique une relation avec d'autres conditions écologiques (sédimentation, courants, transparence de l'eau). Sur le profil de «Procida» se trouvent des éléments coralligènes (surtout ceux d'apect précoralligène), comme Udotea desfontainii, Peyssonnelia squamaria, Eunicella cavolini, et aussi Axinella damicornis, Petrosia ficiformis, Peyssonnelia polymorpba etc., tandis que sur le profil «Posillipo» ne sont présentes qu'en faible quantité certaines Algues précoralligènes, telles que Peyssonnelia squamaria; mais ces éléments dépendent, en premier lieu, de l'ombre qui est plus dense sur la coupe "Procida".

A la station «Mergelina», dont les eaux sont plus polluées, augmente encore le nombre des individus des espèces auxquelles ce biotope convient, comme Mytilus galloprovincialis, Balanus perforatus, Ulva lactuca et Hypnea musciformis; y apparaissent également d'autres espèces adaptées à de telles conditions, telles que Bugula neritina, Zoobotryon verticillatum, Chaetomorpha tortuosa, Bryopsis disticha etc. Les études de Bellan-Santini $(1962,1965)$ sur les peuplements dans les eaux de divers degré de pollution sont très importantes et démontrent la nécéssité de précautions pour l'établissement 
d'indicateurs du degré de pollution de l'eau de mer: Bellan-Santini a trouvé les bancs de Mytilus galloprovincialis très abondants dans deux milieux très differents: dans les zones d'eau polluée (où la teneur des eaux en particules organiques suffisament élevée assure la nourriture à la Moule), ainsi que dans les zones d'eau pure de mode agité (l'agitation de l'eau assure la collecte de la nourriture). La station «Mergelina», par son degré de pollution, correspond aux conditions du peuplement de Mytilus galloprovincialis en eau moyennement polluée, étudié par Bellan-Santini dans le Golfe de Marseille; localement la pollution à la station «Mergelina» devient plus forte.

Les eaux pures de la Méditerranée, étant donnée la quantité limitée de matières nutritives qui les caracterise - oligotrophie - contiennent une biomasse globale relativement faible. Bien qu' y soient représentées des espèces très nombreuses - qui, vu le nombre minime d'individus, représentent souvent une trouvaille rare - les espèces qui sont douées d'une grande capacité d'utilisation du peu de matières nutritives dont elles disposent, prédominent sur le terrain par un nombre important d'individus (par exemple Laurentia papillosa, Cystoseira mediterranea, C. barbata etc., sur le profil de "Procida»). Il est intéressant de constater que ce sont justement ces espèces - par exemple Cystoseira barbata - qui, les plus sensibles à la plus légère pollution (eutrophie), perdant du terrain et cédant la place à d'autres élements qui jusqu' alors étaient plus faiblement représentés, constituent, associés à ces derniers, des communautés instables, disposées en mosaique et d'une production notablement accrue (Golubić, sous presse).

D'un extrême à l'autre, quand les eaux résiduelles menacent plus gravement la biocoenose, le nombre des espèces de la «mosaique» diminue jusqu'à ce que seules quelques espèces subsistent et par leur grand nombre d'individus vigoureux réalisent une haute production. Dans une telle situation apparaissent, d'ordinaire, de nouveaux éléments saprophiles et nitrophiles qui finissent par subsister seuls, comme par exemple la végétation d'Ulva - Hypnea. Allant de ce degré vers la pollution augmentée, le nombre d'individus ainsi que la production totale diminue; la biocoenose autonome fait place aux communautés de bacteries hétérotrophes.

Les végétations de Cyanophycées et de sulfo-bactéries, que Golubic (1960, et sous presse) a étudiées en Adriatique près de Rovinj, représentent une transition entre les biocoenoses complètes et autonomes des eaux marines normales et les communautés dégradées d'eaux marines extrêmement polluées. Quant à la détermination du degré de la pollution des milieux marins les recherches pédologiques de Dufor, Galliano \& Molinier (1960), se basant sur la teneur de l'hydrogène sulfuré et les sulfures dans le sol, sont très significatives. Du point de vue de la santé de l'homme, de l'hygiène et de la thalassothérapie les recherches sur les diverses formes de pollution des eaux marines sont indispensables et urgentes.

\section{RESUME}

1. Le nombre des espèces sténiques réagissant à la plus légère modification du chimisme marin et qui, comme telles, représentent des indicateurs directs - «leitformen" de la qualité de l'eau de mer, est très faible dans le macrobenthos; dans ce domaine 
les recherches microbiologiques et pédologiques apportent une importante contribution.

2. Le passage du biotope oligotrophique au biotope eutrophique a pour conséquences, soit quantitatives, soit qualitatives, soit l'une et l'autre, des changements dans la composition de la biocoenose. L'équilibre de la biocoenose se déplaçant, d'autres espèces deviennent prédominantes. Les indicateurs des eaux oligotrophiques comme Cystoseira barbata, sont remplacés par les indicateurs des eaux eutrophiques, tels que Ulva lactuca, Aplysia fasciata.

3. Les changements quantitatifs (variations du degré d'abondance des espèces) et, en relation avec ceux-ci, le déplacement de l'équilibre de la biocoenose, peuvent servir d'indicateur du degré de pollution de l'eau de mer.

4. La pollution par les eaux résiduelles des biotopes subtidaux est un facteur important, non seulement pour l'étude des lois écologiques générales, mais elle pose aussi un problème de plus en plus pressant des points de vue sanitaire et hygiénique.

Nous adressons ici nos remerciments à la Station Zoologique de Naples qui nous a offert la possibilité de travailler à l'aide d'appareils modernes tant au laboratoire qu'en mer.

\section{LITTÉRATUURE CITÉE}

Bellan-Santini, D., 1962. Etude floristique et faunistique de quelques peuplements infralittoraux de substrat rocheux. Recl Trav. Stn mar. Endoume 26 (41), 237-298.

- 1965. Etude quantitative du peuplement à Mytilus galloprovincialis LamarcK en eau moyennement polluée. Rapp. P.-v. Réun. Commn int. Explor. scient. Mer Méditerr 18, 85-89.

Braun-Blanquet, J., 1951. Pflanzensoziologie. 2. Aufl. Springer, Wien, 631 pp.

Dufour, M., Galliano, J. \& Molinier, R., 1960. Sur l' activité des bactéries sulfato-réductrices dans les sols marins superficiels de la Baie du Brusc (Var). Rapp. P.-v. Réun. Commn int. Explor. scient. Mer Méditerr. 16, 433-437.

ERcegović, A., 1964. Division verticale et horizontale de la végétation des algues adriatiques et ses facteurs. Acta adriat. 19 (9), 75-84.

Gamulin-Brida, H., 1962. Biocoenoses du littoral plus profond (circalittoral) dans les canaux de l'Adriatique moyenne. Acta adriat. 9 (7), 1-196.

- 1965a. Biocoenose des fonds vaseux au large de l'Adriatique moyenne. Acta adriat. 10 (10), $1-27$.

- 1965b. Contribution aux recherches sur la bionomie benthique de la baie de Porto Paone (Naples, Italie). Pubbl. Staz. zool. Napoli, 34, 475-499.

GiacCONE, G., 1965. Le fitocenosi marine nel settore rosso di Capo Zafferano (Palermo). Lav. R. Ist. bot. Palermo 22, 1-69.

- \& Sortino, M., 1964. Flora e vegetazione algale di Isola delle Femmine. Lav. R. Ist. bot. Palermo 21, 140-164.

Golubic, S., 1960. Uber die Blaualgenvegetation in den nordadriatischen Häfen Jugoslawiens. Thalassia jugosl. 2, 5-36.

- 1967. Die Verteilung der Algenvegetation in der Umgebung von Rovinj (Istrien) unter dem Einfluß häuslicher und industrieller Abwässer. Mitt. bayer. biol. VersAnst., München (in press).

Leo, A. DE \& Giaccone, G., 1964. Flora et vegetazione algale del Golfo di Palermo. Lav. $R$. Ist. bot. Palermo 21, 89-120.

Molinier, R. \& Molinier, R., 1955. Eléments de bionomie marine et de phytosociologie aux Iles Sanguinères (Corse). Revue gén. Bot. 62, 675-682. 
Peres, J. M. \& Picard, J., 1958. Manuel de bionomie benthique de la Mer Meditérranée. Recl Trav. Stn mar. Endoume 23 (14), 1-122.

- - 1964. Nouveau manuel de bionomie benthique de la Mer Méditerranée. Recl Trav. Stn mar. Endoume 31 (47), 1-138.

Sara, M. \& Siribelli, L, 1930. La fauna di Poriferi delle «Secche» del Golfo di Napoli. Annuar. Ist. Mus. Zool. Univ. Napoli 12 (3), 1-93.

SORENSEN, T., 1948. A method of establishing groups of equal amplitude in plant sociology based on similarity of species content. K. danske Vidensk. Selsk. $S k r .5(4), 1-34$. 